ฟิ Science and Engineering Research Council

Rutherford Appleton Laboratory

Chilton DIDCOT Oxon OX11 00X

RAL-93-022

\title{
Quark Model Form Factors for Heavy Quark Effective Theory
}

F E Close and A Wambach

May 1993 


\section{Science and Engineering Research Council}

"The Science and Engineering Research Council does not accept any responsibility for loss or damage arising from the use of information contained in any of its reports or in any communication about its tests or investigations" 


\title{
Quark Model Form Factors for Heavy Quark Effective Theory
}

\author{
FE Close \\ Rutherford Appleton Laboratory, \\ Chilton Didcot, Oxon OX11 OQX, England. \\ A Wambach \\ Theoretical Physics, \\ Department of Physics, \\ 1 Keble Road, OXFORD, OX1 3NP, England.
}

\author{
RAL-93-022 \\ OUTP $9306 \mathrm{P}$
}

28.04.1993

\begin{abstract}
We show how both the spectroscopy of heavy-light hadrons and the slope of the Isgur-Wise function can be simultaneously fitted when Heavy Quark Effective Theory is matched onto dynamical quark models, including careful treatment of Wigner rotations. Working consistently to order $\vec{v}^{2}$ and using the parameters from hadron spectroscopy as input, we determine the slope parameter $\rho$ for swave transitions to be $1.13 \pm 0.04$. This agrees with the empirical value for $b \rightarrow c$ transitions.
\end{abstract}




\section{INTRODUCTION}

In recent years considerable experimental and theoretical effort has been invested to understand the physics of hadrons containing a heavy quark. Applications include the determination of important physical quantities such as elements of the KobayashiMaskawa matrix. A fundamental problem for theory is to extract data at quark level from experiments that involve hadrons.

Heavy Quark Effective Theory (HQET) [1] has simplified the analysis by showing that in the limit $M_{Q} \rightarrow \infty$ the spins of the heavy and light degrees of freedom decouple and that a single form factor (the "Isgur-Wise function") describes weak decays of heavy hadrons. In particular this promises that ratios of hadron decays $\Gamma\left(H_{1} \rightarrow\right.$ $\left.\mathrm{H}_{2} l \nu\right) / \Gamma\left(\mathrm{H}_{3} \rightarrow \mathrm{H}_{2} l \nu\right)$ may provide direct measures of the corresponding CKM quark matrix elements $V_{12} / V_{32}$. However, in practice the $M_{Q} \rightarrow \infty$ approximation is bad for strange quarks certainly and arguable for charm. In order to make direct connection between heavy hadron and the corresponding quark amplitudes we need knowledge of the Isgur-Wise function $\xi(y)$ (where $y=v \cdot v^{\prime}$ and $v_{\mu}^{(\prime)}$ is the four-velocity of the heavy meson before (after) the transition), and/or insights into $1 / M_{Q}$ corrections to HQET.

HQET does not predict the $\xi(y)$. The form factor is normalized to unity at the zero recoil point where the meson retains its velocity during the transition. For small, non zero, recoil it is conventional to write

$$
\xi(y)=1-\rho^{2}(y-1)+0\left((y-1)^{2}\right)
$$

where $\rho$ is the "slope parameter" or "charge radius".

There have been several attempts to calculate $\rho$ from theory and models $[2,3,4,5]$, but all appear to underestimate its magnitude compared to the empirical value. A recent calculation of $\xi(y)$ on the lattice appears to be consistent with data, albeit with large uncertainties at present [6].

We shall show that the quark model, when fitted to heavy meson spectroscopy (as in [7]) yields a value of $\rho$ that agrees with form factor data when the operators for current transitions for composite hadrons with recoil are carefully expanded to $0\left(\vec{v}^{2}\right)$. Some previous estimates of $\rho$ in the quark model underestimated it due to an inconsistent non-relativistic approximation.

We begin by reviewing the data and then the existing quark model calculations. The main thrust of the paper is to show how the apparent failure in the latter case 
is due to an incomplete treatment and to demonstrate how the quark model can be consistently applied for heavy-light hadrons.

In refs. $[8,9,10]$ the Isgur-Wise function has been parametrized over a limited range of $y \neq 1$ by making an ansatz for the functional form of its $y$ dependence and comparing this to the available data. The charge radius $\rho$, as defined in eq.(1) is then abstracted from the data.

Refs. $[8,9,10]$ made different ansatze for the functional form of $\xi(y)$ in fitting the data. We rewrite these so that they have a common definition of $\rho$ as in the leading order expansion at eq(1). The different ansatze produce correspondingly slightly different values for $\rho$ with the following results:

$$
\begin{array}{rlrl}
\text { Neubert [8] } & \xi(y)=e^{-\rho^{2}(y-1)} & \rightarrow \rho=1.07 \pm 0.22 \\
{[8,5]} & \xi(y)=\frac{2}{y+1} e^{-\left(2 \rho^{2}-1\right) \frac{y-1}{y+1}} & \rightarrow \rho=1.14 \pm 0.23 \\
\text { (pole ansatz) [8] } & \xi(y)=\left(\frac{2}{y+1}\right)^{2 \rho^{2}} & \rightarrow \rho=1.19 \pm 0.25 \\
\text { Rosner [9] } & \xi(y)=\frac{1}{1+\rho^{2}(y-1)} & \rightarrow \rho=1.29 \pm 0.28 \\
\text { Mannel [10] } \xi(v)=1-\frac{\rho^{2}}{2}(y-1)(y+1) & \rightarrow \rho=0.99 \pm 0.04
\end{array}
$$

Neubert [8] also makes a linear fit to the data:

$$
\xi(v)=1-\rho^{2}(y-1) \rightarrow \rho=0.92 \pm 0.21
$$

On general grounds the universal form factor is expected to have positive curvature for all $y>1$ and so $\rho$ obtained from the linear fit is expected to be a very conservative lower limit [8]. However, the non-relativistic quark model (NRQM) fails even to accomodate this value as we now demonstrate.

In the NRQM where it is assumed that the initial meson is at rest and after the transition moves with the velocity $v^{\prime}=P / M$, the Isgur-Wise function describes the overlap between two light quarks in an s-wave state, where one of the quarks is moving relative to the other. It takes the form:

$$
\xi(v)=\int \phi^{*}\left(k^{\prime}\right) \phi\left(k^{\prime}+m v^{\prime}\right) \mathrm{d}^{3} k^{\prime}=\int \phi^{*}(x) \phi(x) e^{-i m \vec{v}^{\prime} \vec{x}} \mathrm{~d}^{3} x
$$


In the NRQM this comes about because

$$
\phi(p)=\int \mathrm{d}^{3} r_{1} \mathrm{~d}^{3} r_{2} e^{i \vec{p}_{1} \overrightarrow{r i}} e^{i \vec{p}_{2} \overrightarrow{r_{2}}} \phi\left(\vec{r}_{1}-\vec{r}_{2}\right)=\int \mathrm{d}^{3} R \mathrm{~d}^{3} r e^{i \vec{p} \vec{R}} e^{i \overrightarrow{p r}} \phi(r)
$$

that is, the overall momentum $P=p_{1}+p_{2}$ separates itself from the internal momentum $p=\frac{1}{m_{1}+m_{2}}\left(m_{2} p_{1}-m_{1} p_{2}\right)$.

In the ISGW model [7] a Gaussian ansatz $\phi(r)=\exp \left(-\frac{\beta^{2} r^{2}}{2}\right)$ is used to fit the heavy quark spectroscopy. $\beta$ is related to the excitation energy in an harmonic oscillator spectrum. The fit of ref.[7] assumes a linear plus Coulomb potential, and their wavefunctions are treated as a superposition of Gaussians. They require that, if the mass of the degree of freedom represented by the light quark is $330 \mathrm{MeV}$, then $\beta=0.4 \mathrm{GeV}$ for $\mathrm{B}$ and $\mathrm{D}$ hadrons of interest here and $\beta=0.34 \mathrm{GeV}$ for $\mathrm{K}$ systems.

If this wavefunction is used in eq(8) then

$$
\xi(v)=\exp \left(-\frac{\vec{p}^{2}}{4 \beta^{2}}\right) \equiv \exp \left(-\frac{m^{2}}{2 \beta^{2}}(y-1)\right)=1-\frac{m^{2}}{2 \beta^{2}}(y-1)+O\left((y-1)^{2}\right)
$$

This is equal to eq(1) if $\rho^{2}=\frac{m^{2}}{2 \beta^{2}}$. Inserting ISGW values for $m, \beta$ yields $\rho=0.57$, significantly below the experimental value extracted from a Gaussian function (ref[5], eq(2)).Even if one only accepts the result up to the leading term in $(y-1)$ the value of $\rho$ is too small independent of parametrisation chosen in eqs(2)-(7).

Ref.[7] anticipated that their model would be incomplete for large recoil (such as in $b \rightarrow u$ ) and modified the computed slopes of all form factors by a universal multiplicative factor chosen by fitting the observed pion form factor, on the grounds that all heavy-light hadrons have similar size. The effect is that $\rho$ is renormalised [7] $\rho \rightarrow \frac{\rho}{0.7} \approx 0.8$

Even after this ad hoc adjustment the value is only at the lower edge of the value as extracted empirically (refs $[8,5,9,10]$, eqs. (2)-(6) or even eq.(7)). As some of the transitions that are input to the extraction of CKM matrix elements involve non zero recoil it seems desirable to examine this question further.

Ref[11] delineated the kinematic range over which a non-relativistic description of hadron transitions may be consistent, in the sense of preserving the necessary low energy theorems. In ref[12] they showed how these constraints have implications for the matching of heavy quark effective theory and quark model descriptions when applied to current-hadron interactions at $O\left(\overrightarrow{v^{2}}\right)$. They referred to this matching as "Effective 
Heavy Quark Theory" or EHQT. As we shall see in this paper, when the EHQT ideas of ref[12] are applied to the present problem, including the necessary separation of relative and overall momenta variables and consequent Wigner-rotation [12], the value of $\rho$ increases significantly. The step from NRQM to relativistic kinematics increases $\rho$ to about 1 (as has already noted by Neubert [8] following [13], but which does not seem to have been widely appreciated). The approach of "Effective Heavy Quark Theory" - when explicit quark model states are boosted consistently so as to match with Heavy Quark Effective Theory, shows how this relativistic kinematic effect arises but also highlights that Wigner-rotation modifies this further, leading to $\rho=1.13 \pm 0.04$.

The set-up of this paper is as follows. We first briefly repeat the description of meson states in HQET. Then we explain how the Wigner-rotation of the light quark appears in usual quark model descriptions. The next step is to match these two theories and derive a formal representation of heavy mesons from which we can derive the Isgur-Wise function.

\section{MATCHING QUARK MODELS AND HQET}

Ref.[12] showed how to match HQET onto explicit quark model descriptions of current induced transitions. We shall first review this matching in a form that makes contact with the widely used HQET formalism.

An essential assumption is that in the infinite mass limit the heavy quark does not undergo spin interactions with the light degrees of freedom [1]. Interaction with an external field $W_{\mu}$ involves a current $J_{\mu}$ which has the form:

$$
\begin{aligned}
\left\langle D\left(v^{\prime}\right)\left|\overline{Q^{\prime}\left(v^{\prime}\right)} \Gamma_{\mu} Q(v)\right| B(v)\right\rangle & =\overline{Q^{\prime}\left(v^{\prime}\right)} \Gamma_{\mu} Q(v) \times \overline{q^{\prime}\left(v^{\prime}\right)} T\left(v, v^{\prime}\right) q(v) \\
& =\operatorname{Tr}\left[\overline{M^{\prime}\left(v^{\prime}\right)} \Gamma_{\mu} M(v) T\left(v, v^{\prime}\right)\right]
\end{aligned}
$$

where $M(v)=Q(v) \overline{q(v)}$ and $Q(q)$ is the spinor for the heavy (light) quark or antiquark. The coupling for the light quark sector $(T)$ contracts with the mesons' spinwave functions to give a scalar form factor, depending only on $v \cdot v^{\prime}$ (see eg.[14,15]).

Mesons in HQET are therefore described in matrix form. In the rest frame, the matrix description of $s$-wave mesons is written $[15,16]$ :

$$
M(v=(1, \overrightarrow{0}))=\left(\begin{array}{cc}
0 & X \\
0 & 0
\end{array}\right)
$$


where $X=-1$ for the pseudoscalar state $\left(0^{-+}\right)$and $X=\vec{\sigma} \cdot \vec{\epsilon}$ for the vector state $\left(1^{--}\right)$. When boosted to velocity $v_{\mu}=\left(v_{0}, \vec{v}\right)$ one finds for the s-wave mesons:

$$
\begin{aligned}
& M_{0}(v)=\frac{1}{2}(1+\not) \gamma_{5} \\
& M_{1}(v)=\frac{1}{2}(1+\not \gamma)
\end{aligned}
$$

The generalisation to $L \neq 0$ is immediate $[15,14]$.

This is standard. New insights follow when we match this to the quark model by following the prescription of EHQT [12].

For meson transitions it is traditional to use equation (11) as the starting point. But as discussed in refs $[12,17]$ the spinor of the light antiquark has to be defined with care. An example of this can be seen in the work of Golowich et al. [18]. These authors noted that in certain circumstances the neglect of Wigner-rotations associated with recoil in current induced transitions can cause explicit inconsistencies and contradictions in the calculation of form factors for meson decays, in particular $B \rightarrow D^{*} l \nu$ as here. An advantage of the Effective Heavy Quark Theory approach is that such effects are incorporated from the outset and throughout the calculation. We now illustrate this.

If we go to the zero-binding limit, the spinor for the light antiquark in a heavy meson at rest takes the form:

$$
v\left(p_{q}, s_{q}\right)=\sqrt{\frac{\omega_{q}+m_{q}}{2 m_{q}}}\left(\begin{array}{c}
\frac{\vec{\sigma} \cdot \vec{k}}{\omega_{q}+m_{q}} \phi_{q} \\
\phi_{q}
\end{array}\right)
$$

where $\omega_{q}=\sqrt{m_{q}^{2}+\vec{k}^{2}}$ and $m_{q}$ is the mass of the light quark. Now boosting the meson and therefore also the light quark to the velocity $v$ the spinor transforms into

$$
v\left(p_{q}, s_{q}\right)=\sqrt{\frac{1+v_{0}}{2}} \sqrt{\frac{\omega_{q}+m_{q}}{2 m_{q}}}\left(\begin{array}{c}
\left(\frac{\vec{\sigma} \cdot \vec{v}}{1+v_{0}}+\frac{\vec{\sigma} \cdot \vec{k}}{\omega_{q}+m_{q}}\right) \phi_{q} \\
\left(1+\frac{\vec{\sigma} \cdot \vec{v} \cdot \vec{k}}{\left(1+v_{0}\right)\left(\omega_{q}+m_{q}\right)}\right) \phi_{q}
\end{array}\right)
$$

The underlying reason for performing these two boosts (i.e. the $k$ boost followed by the $v$ boost) separately as distinct from directly boosting the light quark to its full four-momentum $\tilde{k}$ is that the rest frame of the meson is the frame where the spins of the heavy and light quark are defined. Therefore one has to set up the description of the meson in this frame, identify the appropriate states in this frame and then boost the whole system to its final velocity $v$ [12]. 
As is obvious from equation (15), if one overlooks the first boost, one fails consistently to account for the Wigner-rotation of the light quark. A similar boost arises for the heavy quark, but at order $1 / M$. This effect for the heavy quark does therefore not appear in the infinite mass limit but has to be included for first order corrections which can be significant, especially when applied to strange quarks in the final state.

To make the matching of HQET and the quark model (thus "EHQT") we return to equation (12). With the matrix description $M=Q \bar{q}$ (or $M=u \bar{v}$ in the quark model language) it is possible to include the light quark boost directly. This then takes the form:

$$
M(v=(1, \overrightarrow{0}))=\left(\begin{array}{cc}
0 & X \\
0 & 0
\end{array}\right)\left(\begin{array}{lr}
1 & -\frac{\vec{\sigma} \cdot \vec{k}}{m_{q}+\omega_{q}} \\
-\frac{\vec{\sigma} \cdot \vec{k}}{m_{q}+\omega_{q}} & 1
\end{array}\right) \sqrt{\frac{m_{q}+\omega_{q}}{2 m_{q}}}
$$

If we scale the light quark momentum and energy by its mass $\left(m_{q}\right)$, then since only the upper right corner in the first matrix is non-zero we can decompose $M$ into an alternative form,

$$
M=\left(\begin{array}{cc}
0 & X \\
0 & 0
\end{array}\right)(1-\not k)[2(1+\omega)]^{-\frac{1}{2}}
$$

Note that we still use $k$ and $\omega$ for the scaled momentum and energy of the light quark to avoid further indices. Using this form and boosting the whole system we derive explicit forms for the $\mathrm{S}$ wave states,

$$
\begin{aligned}
& 0^{-+}=\frac{1}{2}(1+\not \partial) \gamma_{5}(1-\tilde{\not})[2(1+\omega)]^{-\frac{1}{2}} \\
& 1^{--}=\frac{1}{2}(1+\not \partial) \not(1-\tilde{\not})[2(1+\omega)]^{-\frac{1}{2}}
\end{aligned}
$$

Comparing with eqs (13) we see that they are exactly the same meson states but with an additional factor $(1-\tilde{k})[2(\omega+1)]^{-\frac{1}{3}}$ to the right, where $\tilde{k}_{\mu}$ is the boosted four momentum of the light quark (light degrees of freedom) scaled by its mass. These ideas can be generalised to $\mathrm{p}$-waves where again we find the same additional factors [19] arising from the (boosted) relative momenta.

$\mathrm{Eq}(18)$ is essentially a covariant expression for the meson which makes the Wigner rotation of the light quark explicit. It is this form, with explicit appearance of $k$ to the right of $\not p$, that encodes the EHQT in the sense of [12]. The possibility of correlations between the light quark and the heavy quark sector is automatically accomodated if we write mesons in this form; contrast this with many calculations in quark models where one deals with the light and the heavy quark separately. This 
explicit exhibition of such spin correlations will be useful in the next sections when the form factor is evaluated.

In the complete description of the meson wavefunction, the internal momentum distribution $\phi(\vec{k})$ is understood and is normalised such that $\int \mathrm{d}^{3} \vec{k}|\phi(\vec{k})|^{2}=1$. We shall follow the procedure of $\operatorname{ref}[17]$ in which the $\phi(\vec{k})$ factorises from the boost*.

\section{ISGUR-WISE FUNCTION}

We shall now illustrate how these quark model matrices are applied by calculating the Isgur-Wise function to the order ${\overrightarrow{v^{\prime}}}^{2}$ (where $\overrightarrow{v^{\prime}}$ is the velocity of the meson after the transition in the rest frame of the initial meson).

First we make some remarks about the kinematics of the transition.

In the most general case the meson has the four-velocity $v_{\mu}$ before and $v_{\mu}^{\prime}$ after the transition while the light degrees of freedom (which are in the quark model approach synonymous to the light quark) have the four-momentum $\tilde{k}_{\mu}$ before and $\tilde{k}_{\mu}^{\prime}$ after the transition (where we assume throughout that these momenta are scaled by the mass of the light quark). We defined the light quark spinor by starting with an internal momentum $\vec{k}$ and than boosting to the meson velocity. Therefore the usual quark model assumption that the four momenta of the light degrees of freedom are conserved during the transition implies that $\tilde{k}_{\mu}=\tilde{k}_{\mu}^{\prime}$, and hence that

$$
\begin{aligned}
\omega v_{0}+\vec{v} \vec{k} & =\omega^{\prime} v_{0}^{\prime}+\vec{v}^{\prime} \vec{k}^{\prime} \\
\omega \vec{v}+\vec{k}+\frac{\vec{k} \vec{v}}{1+v_{0}} \vec{v} & =\omega^{\prime} \vec{v}^{\prime}+\vec{k}^{\prime}+\frac{\vec{k}^{\prime} \vec{v}^{\prime}}{1+v_{o}^{\prime}} \vec{v}^{\prime}
\end{aligned}
$$

In the rest frame of the decaying meson (i.e. $\left.v_{\mu}=(1, \overrightarrow{0}), v_{\mu}=\left(v_{0}^{\prime}, \vec{v}^{\prime}\right)\right)$ one gets the kinematic constraints:

$$
\begin{aligned}
& \omega=\omega^{\prime} v_{0}^{\prime}+\vec{k}^{\prime} \vec{v}^{\prime} \\
& \vec{k}=\omega^{\prime} \vec{v}^{\prime}+\vec{k}^{\prime}+\frac{\vec{k}^{\prime} \vec{v}^{\prime}}{1+v_{o}^{\prime}} \vec{v}^{\prime}
\end{aligned}
$$

or equivalently

\footnotetext{
*In the absence of this factorisation further renormalisations of the wavefunctions may need to be included -see ref[20].
} 


$$
\begin{aligned}
\omega^{\prime} & =\omega v_{0}^{\prime}-\vec{k} \vec{v}^{\prime} \\
\vec{k}^{\prime} & =-\omega \vec{v}^{\prime}+\vec{k}+\frac{\vec{k} \vec{v}^{\prime}}{1+v_{0}^{\prime}} \vec{v}^{\prime}
\end{aligned}
$$

At $O\left(\vec{v}^{2}\right)$ these already take one beyond the standard non-relativistic formalism and hence these relativistic transformations will modify the calculation of $\rho$ at eq(8). If.one uses $\vec{k} \rightarrow \overrightarrow{k^{\prime}}+\left(\frac{\overrightarrow{v^{\prime}} \vec{k}^{\prime}}{1+v_{0}^{\prime}}+\omega^{\prime}\right) \overrightarrow{v^{\prime}}$ instead of $\vec{k} \rightarrow \overrightarrow{k^{\prime}}+m_{q} \overrightarrow{v^{\prime}}$ in eq (8) the slope parameter turns out to be $\rho=1.03$, an enhancement compared with (3) of about $100 \%$. This has already been noted in [5].

We can understand this result heuristically as follows. In the non-relativistic case we found $\rho^{2}=\frac{m^{2}}{2 \beta^{2}}$ and this becomes $\rho^{2} \simeq \frac{\omega^{2}}{2 \beta^{2}}$ relativistically. Since $\omega^{2} \equiv m^{2}+<\vec{k}^{2}>$ and $\left\langle\vec{k}^{2}\right\rangle \approx O\left(\beta^{2}\right)$ then

$$
\rho_{\text {rel }}^{2} \simeq \frac{m^{2}+\left\langle\vec{k}^{2}\right\rangle}{2 \beta^{2}} \approx \rho_{N R}^{2}+\frac{1}{2} \approx 1 .
$$

However, Wigner rotations of spin have not been accounted for consistently up to this point. The matrix formalism of section 2 shows this explicitly as we shall now demonstrate. This will increase the predicted value for $\rho$ by a further $O(10 \%)$.

Following the approach given above, the s-wave to s-wave transition elements in the unified formulation take the following form:

$$
\begin{aligned}
& \operatorname{Tr}\left[\left(1-\bar{k}^{\prime}\right) L^{\prime} \frac{1}{2}\left(1+\not \not^{\prime}\right) \Gamma_{\mu} \frac{1}{2}(1+\not \partial) L(1-\bar{\not})\right] \phi^{*}\left(k^{\prime}\right) \phi(k) \\
= & \operatorname{Tr}\left[L^{\prime} \frac{1}{2}\left(1+\not \not^{\prime}\right) \Gamma_{\mu} \frac{1}{2}(1+\not) L(1-\overline{\not k}) 2\right] \phi^{*}\left(k^{\prime}\right) \phi(k)
\end{aligned}
$$

where $L\left(L^{\prime}\right)$ is either $\gamma_{5}$ or $\&$ and the normalization factors $(2(1+\omega))^{-\frac{1}{2}}$ are included in the wave functions $\phi$. For the equality we used that $\tilde{k}_{\mu}=\tilde{k}_{\mu}^{\prime}$ and $\tilde{k} . \tilde{k}=1$.

Using this expression, going to the frame defined above with an additional constraint, namely that $\overrightarrow{v^{\prime}}$ is parallel to the z-axis (i.e. $\left.\overrightarrow{v^{\prime}}=\left(0,0, v^{\prime}\right)\right)$, inserting the kinematic constraints (21), the light quark sector contribution to the overlap of initial and final states takes the form:

$$
\int \mathrm{d}^{3} k \mathrm{~d}^{3} k^{\prime}(1-\not k)\left[\left(1+\omega v_{o}^{\prime}-v^{\prime} k_{z}\right)(1+\omega)\right]^{-\frac{1}{2}}\left(\beta_{1} \beta_{2} \pi\right)^{-\frac{3}{2}} e^{-\frac{k^{\prime 2}}{2 \beta_{1}^{2}}} e^{-\frac{k^{2}}{2 \beta_{2}^{2}}} \delta\left(\vec{k}^{\prime}-\vec{k}+\omega \vec{v}^{\prime}-\frac{\overrightarrow{v^{\prime}} \vec{k}}{1+v_{0}^{\prime}}\right)
$$

where we used explicit s-wave quark model wave functions as in [7] and the delta function and normalisation follow the eq(5.8) of ref[17]. 
In the HQET limit $\beta_{1}=\beta_{2}$ but we shall retain different harmonic oscillator strengths here because in practice for charm and strange quarks, with non-infinite masses, these strengths differ considerably (see [7]). Expanding the exponential and the inverse square root in $v^{\prime}$ and keeping only terms proportional to $v^{\prime}$ and $v^{\prime 2}$ one derives

$$
\begin{gathered}
\left(\pi \beta_{1} \beta_{2}\right)^{-\frac{3}{2}} \int \mathrm{d}^{3} k\left[\left(1-\omega \gamma_{0}+\vec{k} \vec{\gamma}\right)(1+\omega)^{-1} e^{-A k^{2} \times}\right. \\
\left.\left(1+v^{\prime} k_{z}\left(\frac{1}{2(1+\omega)}+\frac{\omega}{\beta_{1}^{2}}\right)+v^{\prime 2}\left(\frac{-\omega}{4(1+\omega)}-\frac{\omega^{2}}{2 \beta_{1}^{2}}+k_{z}^{2}\left(\frac{3}{8(1+\omega)^{2}}-\frac{\omega}{2 \beta_{1}^{2}(1+\omega)}-\frac{1}{2 \beta_{1}^{2}}+\frac{\omega^{2}}{2 \beta_{1}^{4}}\right)\right)\right)\right]
\end{gathered}
$$

$A$ is defined to be $\frac{1}{2}\left(\beta_{1}^{-2}+\beta_{2}^{-2}\right)$. Before we complete the calculation, note first the structure of the expression in (24). It is obvious that the final result will have the form:

$$
B+C \gamma_{0}+v^{\prime} D \gamma_{z}=B+\left(C+v_{0}^{\prime} D\right) \not \partial-D \not \gamma^{\prime}
$$

where on the RHS we specialised to the frame $v=(1,0,0,0)$ and $v^{\prime}=\left(v_{0}^{\prime}, 0,0, v^{\prime}\right)$. Because at the end of the calculation we have to insert this expression into (22) we now can contract $\not p$ and $\not^{\prime \prime}$ with $\left(1+\not \not^{\prime}\right)$ and $\left(1+\not \not^{\prime}\right)$. This has the consequence that the Isgur-Wise function in this quark model approach takes the form:

$$
\xi(v)=B-\left(C+v_{0}^{\prime} D\right)+D=B-C-\frac{1}{2} v^{\prime 2} D
$$

This has the effect that

$$
1-\omega \gamma_{0}+k_{z} \gamma_{z} \rightarrow 1+\omega-\frac{1}{2} v^{\prime} k_{z}
$$

At this stage the relativistic form of a heavy meson in the quark model developed in (16),(18) reduces the calculation for the light quark sector considerably, because of the direct contraction with the heavy quark sector. Doing the calculation one gets for the form factor:

$$
\begin{gathered}
\xi\left(v^{\prime}\right)=\left(\frac{\beta_{1}^{2}+\beta_{2}^{2}}{2 \beta_{1} \beta_{2}}\right)^{-\frac{3}{2}}\left(1+v^{\prime 2}\left[-\frac{1}{2} \beta_{1}^{-2}-A^{-1}\left(\beta_{1}^{-2}-\frac{1}{4} \beta_{1}^{-4}\right)+\frac{5}{8} A^{-2} \beta_{1}^{-4}\right]\right) \\
+v^{\prime 2}\left(\pi \beta_{1} \beta_{2}\right)^{-\frac{3}{2}} \int\left(\frac{-\omega}{4(1+\omega)}+\frac{k_{2}^{2}}{8(1+\omega)^{2}}\right) e^{-A k^{2}} \mathrm{~d}^{3} k
\end{gathered}
$$

where $A$ is defined as in (24). This leads to $\rho=1.17$ where we fitted the harmonic oscillator strength with the value given by [7]. 
Relativistic recoil and Wigner rotation of the light quark have each increased the slope of the Isgur-Wise function. The non-relativistic quark model gave $\rho=0.57$; taking account of relativistic kinematics for the light quark while ignoring spininteraction increased this to $\rho=1.03$ and including a rotation of the light quark spin increases $\rho$ to 1.17 . This qualitative behaviour is quite reasonable. The Wigner spin rotation effectively decreases the probability for the final state pseudoscalar to overlap with the initial and hence the form factor falls faster, or equivalently $\rho$ increases, than when this effect is ignored.

One of the problems encountered in NRQM calculations is that in general the results depend on the choice of frame. In the present example the calculation of the Isgur-Wise function in the rest frame of the decaying meson or in the rest frame of the final meson is the same. However the result is slightly changed if the calculation is done in the 'Breit-frame'. The source of this frame dependence is that in the former calculation one meson is always at rest, while in the 'Breit-frame' both mesons are moving. This means that for the light quark before and after the transition we have to include the two boosts, namely first to the internal momentum $\vec{k}\left(\overrightarrow{k^{\prime}}\right)$ and then to the overall momentum $\vec{P} / M\left(\vec{P}^{\prime} / M^{\prime}\right)$. We therefore state as our final result for the charge radius the average of both calculations: $\rho=1.13 \pm 0.04$.

In general (22) allows the calculation of the form factor to an arbitrary order in $v^{\prime}$, but the quark model loses much of its predictive power when $y>>1$ because the assumption $\tilde{k}_{\mu}=\bar{k}_{\mu}^{\prime}$ is no longer justified. As pointed out in [14] gluon exchange with the heavy quark at the transition vertex can give additional momentum to the light quark (light degrees of freedom) after the transition, (the so-called velocity-kick [21]), which goes beyond the approximation of the present paper.

\section{CONCLUSIONS}

The authors of ref.[12] showed how to match quark model and HQET in the particular case of electromagnetic transitions and where all interactions between the constituents were ignored. In [12] a non-trivial structure emerged as a result of dealing carefully with the Wigner-rotation. The essential features generalized to the case of present interest and enabled a compact description of mesons suitable for EHQT (eq18).

We applied these ideas to an explicit calculation of the form factors for heavy flavour transitions involving S-wave states. We found that the calculations of the 
non-relativistic ISGW model (ref [7]) are changed significantly and that the effect of Wigner rotations merits care.

In particular we found that the model parameters required to fit the spectroscopy [7] lead to an excellent description of the slope of the universal form factor, in contrast to previous literature. Specifically, in this quark model calculation the $\rho$ parameter which is defined via $\xi(y)=1-\rho^{2}(y-1)+\mathrm{O}\left((y-1)^{2}\right)$ turned out to be:

$$
\rho=1.13 \pm 0.04
$$

This describes an enhancement of the effect that the light degrees of freedom.have compared with the simple quark model calculation which gave $\rho=0.568$ and the relativistic calculation without Wigner rotation: $\rho=1.03$. This result flowed from the expansion of the current operator to $O\left(\vec{v}^{2}\right)$ as required for consistency.

It is interesting to note the parallel with similar work in the light quark sector where Close and $\mathrm{Li}$ [11] pointed out that the apparent difference between parameters for fitting spectroscopy [22] and current induced transitions ( $\gamma$ and electroproduction) were artefacts of an inconsistent non-relativistic restriction. When calculations of current transitions were made consistently to $O\left(\overrightarrow{v^{2}}\right)$ both dynamics and spectroscopy could be simultaneously described [11].

Our calculations highlight the role of the two independent $(\vec{k}, \vec{v})$ boosts. On physical grounds we expect that these considerations are more general than the specific model. However, in turn, the present approach has its limitations. We have shown that the expectation of current operators between spinors of massive quarks in composite systems leads to significant deviations from naive NRQM but, as shown in ref [11], the binding potential can also make an explicit contribution to the current operator. The major effect from a scalar potential is to renormalise the quark mass; insofar as this is a parameter in the model, it is effectively incorporated in the present work. The question of the factorisation of $\phi(\vec{k})$ from the overall boost merits further investigation (see also ref[20]).

Our calculations provide a measure of the validity of Heavy Quark Effective Theory in finite mass situations. The Wigner spin rotations arise when boosting a state whose constituents $\frac{\vec{k}}{m} \neq 0$ in the overall rest frame. For b-quarks this effect is less than $1 \%$ and can be ignored: HQET applies. For c-quarks the effect of $\left(\frac{\vec{k}}{m}\right)^{2}$ is of order up to $10 \%$ and so caution should be exercised at this level. For strange quarks this term is of the order $30 \%$ and can not be ignored. This deviation from the infinite mass limit shows itself e.g. in the mixing of the ${ }^{3} P_{1}$ and ${ }^{1} P_{1}$ states in the 
Kaon-system, where in the heavy mass limit one would expect a mixing angle of $35^{\circ}$ [21], while experiments indicate an angle of about $45^{\circ}$ [23]. This may modify some of the analysis of $B \rightarrow K^{* *} \gamma$ [4]. This entails extension of these ideas to P-states and is under investigation [19].

We are indebted to A.Le Yaouanc and J.C.Raynal for helpful comments. A.W. thanks the German Academic Exchange Service and the German Scholarship Foundation for financial support.

\section{References}

[1] N. Isgur and M.B. Wise, Phys. Lett. B 237 (1990) 527

[2] B. Blok and M. Shifman, NSF-ITP-92-100

[3] M. Voloshin, Phys. Rev. D 46 (1992) 3062

[4] A. Ali, T. Ohl and T. Mannel, Phys. Lett. B298 (1993) 195

[5] M. Neubert and V. Rieckert, Nucl. Phys. B 382 (1992) 97

[6] UKQCD-collaboration, private communication and preprint Edinburgh $93 / 525$; Southampton SHEP 92/93-17

[7] N. Isgur, D. Scora, B. Grinstein, M. Wise, Phys. Rev. D 39 (1989) 799

[8] M. Neubert, Phys. Lett. B 264 (1991) 455

[9] J. L. Rosner, Phys. Rev. D 42 (1990) 3732

[10] T. Mannel, W. Roberts, Z. Ryzak, Phys. Lett. B 254 (1991) 274

T. Mannel, IKDA $92 / 6$

[11] F. E. Close and Z. Li, Phys. Rev. D 42 (1990) 2194

F. E. Close and Z. Li, Phys. Rev. D 42 (1990) 2207

[12] F. E. Close and Z. Li, Phys. Lett. B237 (1992) 143

[13] M.Wirbel, B.Stech and M.Bauer, Z.Phys C29, (1985) 637 
[14] S. Balk, J. G. Körner, G. Thompson, F. Hussain, IC-91-397, June 1992

[15] A. F. Falk, Nucl.Phys. B 378 (1992) 79

[16] J. D. Bjorken, invited talk given at "Les Recontre de la Valle d'Aosta La Thuile" Editions Frontiers, Gif-Sur-Yvette:(1990) 583

[17] S. Brodsky and J. Primack, Ann. Phy. 52, 315 (1969)

[18] E. Golowich et al., Phys. Lett. B 213 (1988) 521

[19] A.Wambach, in preparation

[20] A.LeYaouanc et al., Ann. Phy. 88, 242 (1974) and Erratum 97, 567 (1976). A.LeYaouanc et al.,Phys. Rev. D 15 (1977) 844

[21] J. G. Körner, Nucl.Phys. B (Proc. Suppl.) 21 (1991) 366

[22] N. Isgur and G. Karl, Phys. Rev. D 18 (1979) 4187 N. Isgur and G. Karl, Phys. Rev. D 19 (1979) 2653

[23] G. W. Brandenburg et. al., Phys. Rev. Lett. 36 (1976) 703 R. K. Carnegie et. al., Phys. Lett. B 68 (1977) 287 


